\title{
WHAT WE CAN READ AND WHAT WE CANNOT READ IN THE MORTALITY CURVE OF THE NEONATAL SURGERY THE PROGRESS OF THE NEONATAL SURGERY IN JAPAN
}

The progress of neonatal surgery was reviewed from the viewpoint of the mortality curve (MC) based on the nationwide survey of neonatal surgery, which has been conducted by Japanese Society of Pediatric Surgeons once every 5 years since 1964 . The mortality of major diseases was over $60 \%$ in 1960 s, which has decreased to $20 \%$ or less for the last 50 years. The contributing factors in this drastic decrease must include progress of neonatal medicine, such as respiratory care, concentration of the cases, induction and spread of parenteral nutrition (PN) and education of pediatric surgeons (PS) through annual meetings of JSPS, specialist certification system, set-up of pediatric surgical sections in medical schools or children's hospitals. The MC of intestinal atresia turned to rapid declining after the first success of PN in 1968 followed by rapid spread. The MC of congenital diaphragmatic hernia went upward at the first 4 surveys since 1973. This paradoxical shift could be explained by early diagnosis and prompt transportation to PS, or the more $\mathrm{CDH}$ were diagnosed earlier, the more sever or lethal cases were treated by PS. The MC of intestinal perforation (IP) turned to increasing in 1993 after long-term steady decline, because the more extremely-low-birth-weight newborns were saved, the higher incidence of IP characteristic of ELBWs was noticed. Omphalocele is the only one disease which MC showed increase for the last five years, which

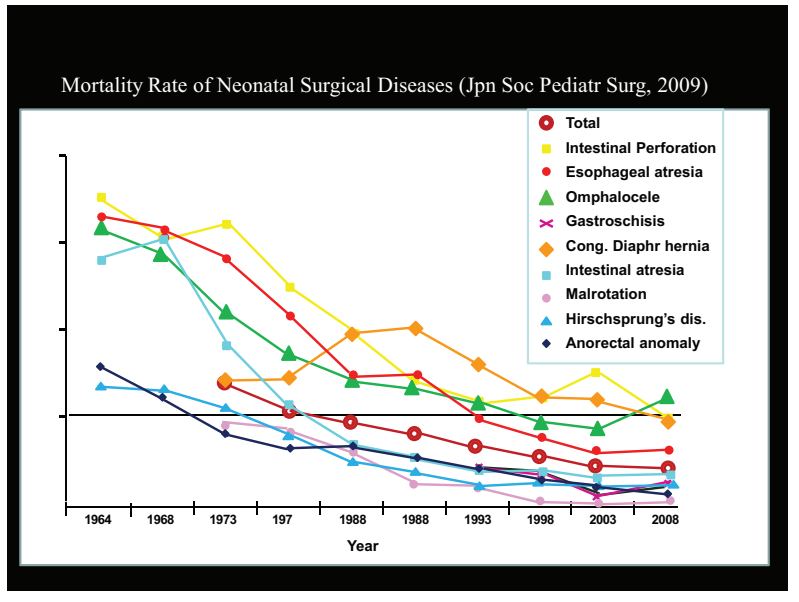

may be explained by the ethical consideration for antenatally diagnosed serious cases. A case of esophageal atresia was saved for the first time in 1960, or the mortality was $100 \%$ until that time, however, the MC declined to 60 s $\%$ by 1964 , and to $11 \%$ by 2008, due to all the effort by PS in Japan. Meanwhile, unfortunately, the MC cannot tell anything about postoperative functional achievement, mental development and long-term QOL.

Akio Kubota, MD, PhD

Professor of Pediatric Surgery, Second Department of Surgery, Wakayama Medical University, Wakayama, Japan, kubota@wakayama-med.ac.jp 